\title{
Measurement of Antennas and Microwave Components Using Time-Domain Reflectometry of a Voltage Impulse
}

\author{
Steven McCabe, Student Member, IEEE, and Jonathan Scott, Senior Member, IEEE
}

\begin{abstract}
Band-pass microwave systems such as ultrawideband (UWB) antennas are traditionally characterized in the frequency-domain through a vector network analyzer (VNA) in an anechoic chamber. A recent study proved antennas could be accurately measured in the time-domain using a step-function time-domain reflectometer (TDR), without the need for an anechoic chamber. We propose a new advance in the TDR characterization method. An impulse generator is employed in place of the step generator in a TDR set-up. The advantage conferred by this change is that more energy is available beyond a given frequency than with a step, and so a higher signal-tonoise ratio (SNR) is achieved. The theoretical result is compared with measurement.
\end{abstract}

Index Terms-Antenna measurements, frequency-domain (FD) analysis, pulse measurements, time domain reflectometry (TDR), transient response.

\section{INTRODUCTION}

$\mathbf{T}$ IME-DOMAIN reflectometry (TDR) is a well-known technique in electronic and optical systems [1]-[4]. It has recently been used successfully in the characterization of antennas in [5] and [6]. The motivation behind this is twofold: A TDR is less costly than a vector network analyzer (VNA) [5], but more importantly the time-localization of the energy in the test signal means that the user can dispense with the anechoic chamber that is required for antenna measurements with a sinewave exciting signal.

The traditional stimulus signal in a TDR is a voltage step. The energy in a step signal falls with increasing frequency. In the situation where the device under test (DUT) is interesting in only a limited high bandwidth, as with antennas, most of the energy of the step is wasted and the dynamic range of the measurement is limited. On the other hand, an impulse test signal has a theoretically flat bandwidth. In this paper, we explore the advantages of making impulse TDR measurements, similar to a traditional TDR but employing an impulse-like signal instead of a step-like signal. The work is timely because a high-quality impulse generator has become available [7]-[9]. This allows us to compare theory with measurement.

\section{Fourier Transform of the Stimulus Signal}

The unit impulse (Dirac delta) function is defined as having zero amplitude for all time except at $t=0$, where it has infinite amplitude:

$$
\delta(t)= \begin{cases}0, & t \neq 0 \\ \infty, & t=0\end{cases}
$$

The Fourier transform for the unit impulse is

$$
\mathrm{F}[\delta(t)]=1
$$

An ideal impulse has a flat frequency response. Although the unit impulse is a theoretical construct and does not physically exist [10], it is used as a limiting case when the width of a pulse approaches zero. Derived from the convolution of two rectangular ("rect") functions, the trapezoid function provides an approximation of a realistic impulse with finite rise and fall times [11]:

$$
u(t)=\frac{1}{\tau} \operatorname{rect}\left(\frac{t}{\tau}\right) \otimes A \operatorname{rect}\left(\frac{t}{T}\right)
$$

where $A$ is the trapezoid amplitude, $T$ is the full width at half maximum (FWHM), and $\tau$ is the rise/fall time from 0 to $100 \%$ of the amplitude. The Fourier transform of $u(t)$ is given by

$$
\mathrm{F}[u(t)]=A T \operatorname{sinc}(f \tau) \operatorname{sinc}(f T)
$$

The Heaviside unit step function is defined as

$$
H(t)= \begin{cases}1, & t>0 \\ \frac{1}{2}, & t=0 \\ 0, & t<0\end{cases}
$$

This function represents an ideal voltage step which is immediately elevated to a constant level at a definite time [12]. The Fourier transform of $H(t)$ is given by

$$
\mathrm{F}[H(t)]=\frac{1}{j 2 \pi f}+\frac{1}{2} \delta(f)
$$

The response varies as the reciprocal of frequency and so approaches zero amplitude as frequency tends to infinity. This theoretical construct does not physically exist because a realistic step waveform has a finite rise time. The Fourier transform for a step function $s(t)$ with finite rise time is given by

$$
\mathrm{F}[s(t)]=\frac{1}{j 2 \pi f} \operatorname{sinc}(f \tau)
$$

where $\tau$ is the rise time from 0 to $100 \%$ of the amplitude. Fig. 1 presents a number of spectra that will be compared 


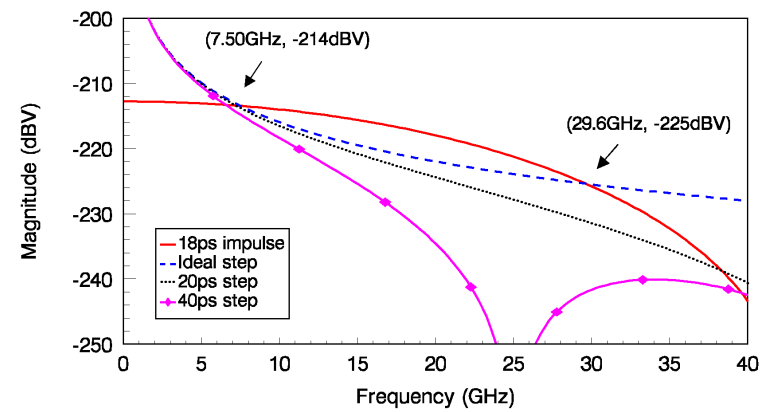

Fig. 1. The Fourier transforms of an ideal unit step, some practical steplike signals, and a practical trapezoidal pulse of unit amplitude. The unit for magnitude is $\mathrm{dBV}$, namely the voltage relative to 1 Volt.

with the spectrum of an ideal step (Heaviside unit step) that appears as the dashed line. The new impulse source generates impulses with a typical FWHM of 23 ps [13]. The rise/fall time is estimated as $18 \mathrm{ps}$ ( 5 ps flat-top). By inserting these parameters in (3), the Fourier transform of a unit amplitude impulse was simulated in Matlab. This function can now be compared with the unit step from (4) and some realistic step waveforms from (5) in Fig. 1. It can be seen that even the realistic, limited impulse signal contains more energy than an ideal step for frequencies above about $7.5 \mathrm{GHz}$. When the step waveform is not ideal, but similar to what is practically available today, the comparison becomes even more favorable, as can be seen in the same figure.

\section{MEASURED RESUltS}

An impulse-style TDR was constructed as shown in Fig. 2. The set-up features an impulse generator with a $23 \mathrm{ps}$ pulse width [7]-[9], a DC to $26.5 \mathrm{GHz}$ power divider, and an $18 \mathrm{GHz}$ TDR operating in oscilloscope mode [14]. The DUT was a Wide-Band sector antenna designed to operate between 5.47-5.85 GHz. A step-type TDR set-up was constructed using a similar configuration but with the impulse source replaced with a $50 \Omega$ dummy load and the mainframe set in TDR mode. The divider was included to replicate the conditions of the impulse TDR set-up so that fair comparisons between techniques could be made. An attenuator was also inserted between the oscilloscope channel and the divider, to further reduce the step signal to a similar amplitude produced in the impulse TDR set-up. Calibrated measurements were acquired using a VNA, where the DUT was situated outdoors to provide an accurate reference for comparison with the TDR measurements.

\section{A. Acquisition in the Time-Domain}

Two time-domain measurements were employed in the subsequent frequency-domain processing: A reference waveform reflected from a short, and the waveform reflected by the antenna. Averaged measurements taken by the impulse TDR set-up were windowed with the Dirichlet window in Matlab, to remove the incident portion of the signal and to remove spurious reflections as required for subsequent frequencydomain processing as reported in [5]. The rectangular window contained the essential data that corresponded to the DUT

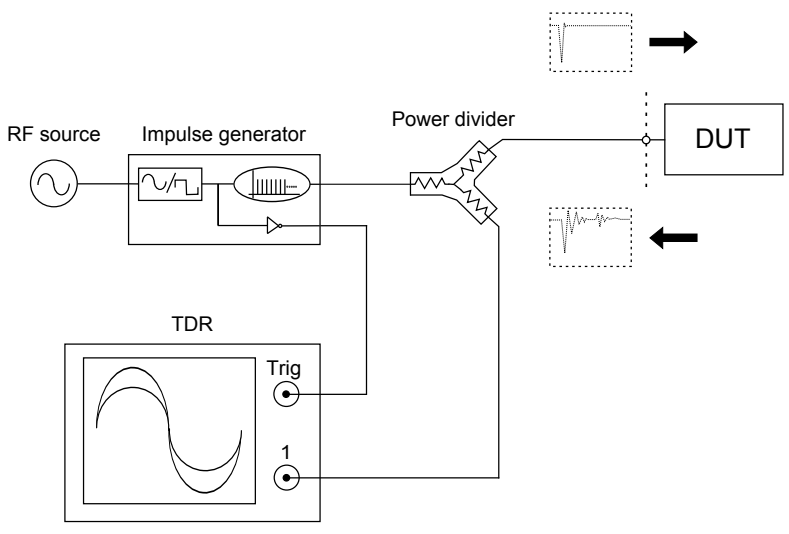

Fig. 2. An impulse TDR set-up for the measurement of microwave devices. A generated impulse is transferred to the DUT, resulting in a reflection that is monitored on the TDR. The dashed line represents the reference plane at the DUT connector.

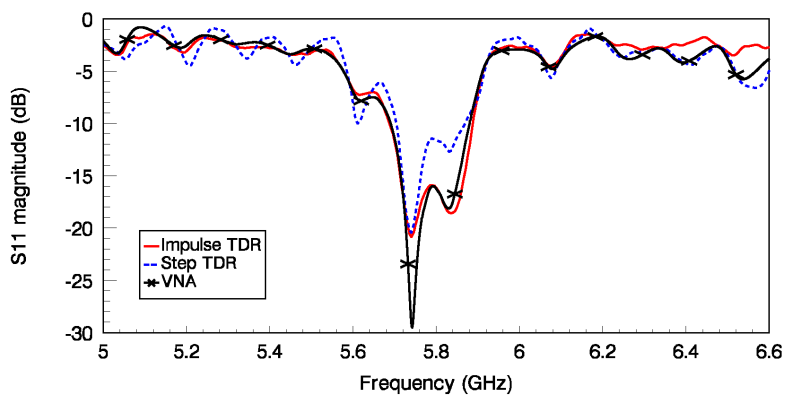

Fig. 3. Comparison of $\left|S_{11}(f)\right| \mathrm{dB}$ measurements made by the impulse TDR, step TDR, and VNA, limited to the working frequency range of the wide-band antenna. The superior dynamic range of the VNA is evident where the magnitude drops to almost $-30 \mathrm{~dB}$.

response, and provided a reference plane extended to the end of the cable where the DUT was to be attached. The length of the time window was chosen so that there was sufficient time for the antenna's reflection to settle, while limited to block out spurious reflections caused by the presence of objects near the antenna. Initially, a 35 ns acquisition window was chosen as endorsed in [5]. The same process was followed for the acquisition of the step TDR measurements.

\section{B. Transformation to the Frequency-Domain}

The zero-padding operation was employed to ensure adequate frequency resolution, along with the application of the Nicolson algorithm to avoid truncation error [3]. A computer program computed the discrete Fourier transform (DFT) for both the reference reflection and DUT reflection, resulting in $V_{\text {ref }}(f)$ and $V_{D U T}(f)$. The reflection scattering parameter $S_{11}(f)$ was then computed by $S_{11}(f)=V_{D U T}(f) / V_{\text {ref }}(f)$ for frequencies between 2.0-9.0 GHz. The DUT $\left|S_{11}(f)\right| \mathrm{dB}$ response obtained by both the impulse TDR and step TDR methods, were plotted against the VNA reference as shown in Fig. 3. The frequency range shown is constrained to the antenna's specified operating range.

\section{Variance}

A plot of the $\left|S_{11}(f)\right|$ variance measured between the TDR measurements and the VNA reference are shown in Fig. 4. 


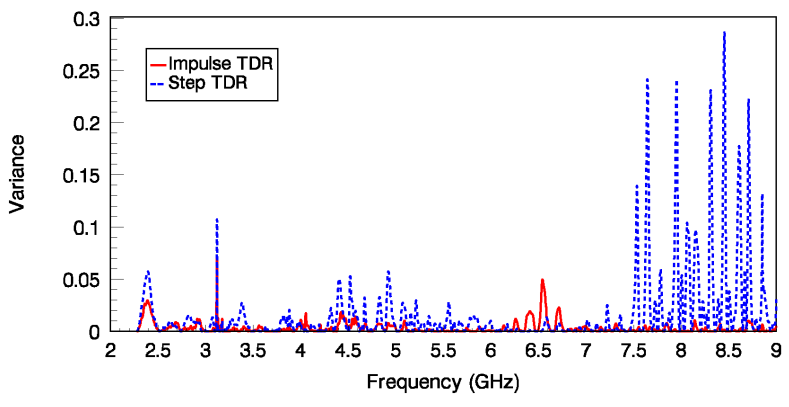

Fig. 4. $\left|S_{11}(f)\right|$ variance measured to $9 \mathrm{GHz}$ between each TDR system response and the VNA reference.
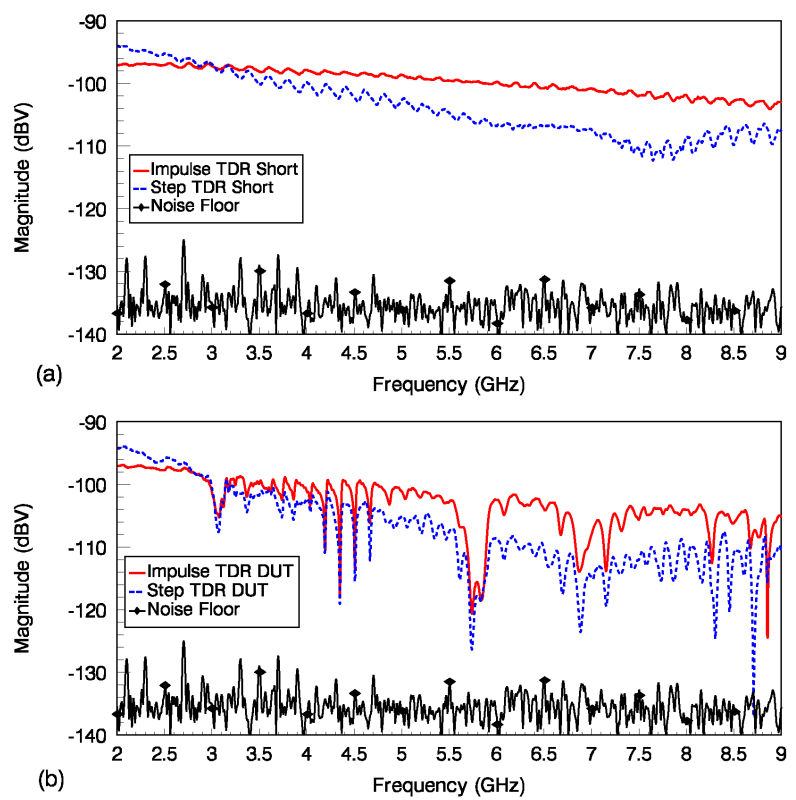

Fig. 5. The frequency spectra for the impulse TDR and step TDR measurements when the (a) reference short was connected at the reference plane (b) DUT was connected at the reference plane. The noise floor was determined by measuring the noise present in the system with the signal source disabled.

The frequency axis is limited to the $9.0 \mathrm{GHz}$ bandwidth of the VNA. The impulse TDR measurement clearly contains less variance than the step TDR measurement, especially above $7.0 \mathrm{GHz}$.

\section{Dynamic Range}

The spectra measured in the reference short reflections for both the impulse TDR and step TDR systems are shown in Fig. 5(a). Similarly, the spectra present in the DUT reflection for each TDR set-up are shown in Fig. 5(b). It is apparent from both figures that the energy in the impulse TDR reflection exceeds the energy in the step TDR reflection above about $3.1 \mathrm{GHz}$. Beyond $3.1 \mathrm{GHz}$ the $23 \mathrm{ps}$ impulse TDR offers a significant improvement in SNR compared with the Agilent 54754A 40 ps step TDR. Recommendations for the application of impulse TDR, step TDR, and VNA measurement techniques, are summarized in Table I. The numerical value for each parameter will vary with circumstance and so only trends are given.
TABLE I

COMPARISON OF MEASUREMENT TECHNIQUES

\begin{tabular}{|c|c|c|c|c|}
\hline Technique & $\begin{array}{c}\text { Bandwidth } \\
\text { (GHz) }\end{array}$ & $\begin{array}{c}\text { Dynamic } \\
\text { range }\end{array}$ & $\begin{array}{c}\text { Equipment } \\
\text { cost }\end{array}$ & $\begin{array}{c}\text { Anechoic } \\
\text { chamber }\end{array}$ \\
\hline Impulse TDR & Med & Med & Med & No \\
Step TDR & Low & Low & Med & No \\
VNA & High & High & High & Yes \\
\hline
\end{tabular}

\section{CONCLUSION}

In the situation where the user will post-process reflection waveform data, for example, to determine S-parameters, the impulse version of TDR is superior at frequencies above $7 \mathrm{GHz}$. It may also be advantageous when the user is looking for precision in spatial localization, say in a connector or similar in-line structure, as the increased energy at higher frequencies can help.

\section{ACKNOWLEDGEMENT}

The authors wish to acknowledge the support of Agilent Technologies Component Test Division for the supply and detailed support of equipment. Thanks are also due to Skynet Data Communications Technology Ltd for the supply of equipment and support.

\section{REFERENCES}

[1] C. Chiu, W. Chen, K. Liao, B. Chen, Y. Teng, G. Huang, and L. Wu, "Pad characterization for CMOS technology using time domain reflectometry", in Proc. IEEE Int. RF and Microwave Conf., in Kuala Lumpur, Dec. 2008, pp. 215-217.

[2] M. Harper, N. Ridler, and M. Salter, "Comparison between root-impulseenergy and vector network analyzer methods for measuring loss on printed circuit boards", in ARFTG Microwave Measurement Symp., in Portland, OR, 2008, pp. 20-25.

[3] A. Cataldo, L. Catarinucci, L. Tarricone , F. Attivissimo, and A. Trotta, "A TD-FD combined method for enhancing reflectometry measurements in liquid quality monitoring", in Proc. IEEE Instrum. Meas. Tech. Conf., in Warsaw, May. 2007, pp. 1-5.

[4] M. Gerding, T. Musch, and B. Schiek, "A novel approach for a highprecision multitarget-level measurement system based on time-domain reflectometry" IEEE Tran. Microw. Theory Tech., vol. 54, no. 6, pp. 2768-2773, Jun. 2006.

[5] A. Cataldo, G. Monti, E. De Benedetto, and G. Cannazza, L. Tarricone, and L. Catarinucci, "Assessment of a TD-based method for characterization of antennas", IEEE Trans. Instrum. Meas., vol. 58, no. 5, pp. 1412-1419, May. 2009.

[6] R. Tamas, G. Caruntu, and D. Popa, "A time-domain measuring technique for ultra-wide band antennas", Microwave and Optical Technology Letters, vol. 53, no. 2, pp. 281-286, Feb. 2011.

[7] J. Scott and D. Gunyan, "Pulse generator", US patent number 7,423,470, Sep, 2008.

[8] J. Scott and M. Hoy, "Group-delay measurement of frequencyconverting devices using a comb generator", IEEE Trans. Instrum. Meas., vol. 59, no. 11, pp. 3012-3017, Nov. 2010.

[9] P. Blockley, D. Gunyan, and J. Scott, "Mixer-based, vector-corrected, vector/network analyzer offering $300 \mathrm{kHz}-20 \mathrm{GHz}$ bandwidth and traceable response", IEEE Int. Microwave Symp. in Long Beach, CA, Jun. 2005, pp. 1-4.

[10] S. Haykin and M. Moher, Introduction to analog \& digital communications, second edition, John Wiley \& Sons, Inc, pp. 42-49, 2007.

[11] D. Brandwood, Fourier transforms in radar and signal processing, Artech House, Inc., pp. 39-47, 2003.

[12] R. N. Bracewell, The fourier transform and its applications, third edition, McGraw-Hill International Editions, pp. 61, 2000.

[13] Agilent U9391C/F comb generators technical overview, Agilent Technologies, [Online]. Available: http://www.home.agilent.com/agilent/ product.jspx?pn=U9391C retrieved Jan 2010.

[14] Agilent 54753A and 54754A TDR plug-in modules user guide, Agilent Technologies, publication number 54753-97015, 2000. 